\title{
POLARIZATION OF $\Sigma^{0}$ HYPERONS IN INCLUSIVE PRODUCTION FROM 28.5 GeV/c PROTONS ON BERYLLIUM
}

\author{
Edmond C. DUKES ', Oliver E. OVERSETH \\ Department of Physics, University of Michigan. Ann Arbor, MI 48109, USA
}

Gerry M. BUNCE, Yousef MAKDISI, Peter YAMIN

AGS Department, Brookhaven National Laboratory, Associated Universities, Inc., Upton, Long Island, NY 11973, USA

\author{
Douglas A. JENSEN, Michael N. KREISLER, Martin MARCIN ${ }^{2}$, Kamal K. RAYCHAUDHURI ${ }^{3}$, \\ Marilyn W. SULLIVAN ${ }^{3}$ \\ Department of Physics and Astronomy, University of Massachusetts, Amherst, MA 01003, USA
}

Kenneth HELLER and Mike SHUPE

School of Physics and Astronomy, University of Minnesota, Minneapolis, MN 55455, USA

Received 19 March 1987

\begin{abstract}
The polarization of $\Sigma^{0}$ hyperons produced in an inclusive reaction has been measured for the first time. From a sample of 11000 events produced by $28.5 \mathrm{GeV} / c$ protons in the reaction $\mathrm{p}+\mathrm{Be} \rightarrow \Sigma^{0}+\mathrm{X}$, the $\Sigma^{0}$ polarization has a value of $+0.28 \pm 0.13$ at $p_{1}=1.01 \mathrm{GeV} / c$ and $x_{\mathrm{f}}=0.60$. The polarization of $53000 \Lambda$ hyperons produced from $28.5 \mathrm{GeV} / c$ protons in the reaction $\mathrm{p}+\mathrm{Be} \rightarrow \Lambda+\mathrm{X}$ has also been measured in the kinematic range $0.64<p_{\mathrm{t}}<1.14 \mathrm{GeV} / c$ and $0.42<x_{\mathrm{f}}<0.62$. The average $\Lambda$ polarization is found to be $-0.188 \pm 0.024$, consistent with previous results.
\end{abstract}

Since the discovery of large polarization in inclusive $\Lambda$ production at $300 \mathrm{GeV}$ in 1976 [1] further experimental effort has been directed toward both expanding the knowledge of the kinematic dependence of the polarization and searching for similar behaviour in the inclusive production of other hadrons. These efforts have yielded measurements of the polarization in inclusive production of all the hyperons except the $\Sigma^{0}$. Polarization has been found not to be unique to the $\Lambda$, but in fact a universal feature in inclusive hyperon production from incident protons, with the $\Lambda, \Sigma^{+}, \Sigma^{-}, \Xi^{-}$, and $\Xi^{0}$ hyperons all

1 Present address: EP Division, CERN, CH-1211 Geneva 23, Switzerland.

2 Present address: Department of Physics, Rice University, Houston, TX 77001, USA.

3 Present address: AT \& T Bell Laboratories, Holmdel, NJ 07733, USA. exhibiting substantial polarization ${ }^{\# 1}$. The kinematic behavior of these polarizations is quite similar with the exception of the sign of the polarization. The $\Sigma^{+}$ and $\Sigma$ - hyperons are produced with positive polarization (defined to be in the $\boldsymbol{p}_{\text {beam }} \times p_{\text {hyperon }}$ direction) whereas the $\Lambda, \Xi^{-}$, and $\Xi^{0}$ ajre found to be negatively polarized. We report here the first measurement of the polarization of inclusively produced $\Sigma^{0}$ hyperons.

The experiment (E744) was situated in the B5 beam line at the AGS at Brookhaven National Laboratory. Since it is known that inclusive polarization is not strongly dependent on energy, we chose to do this experiment at an energy where the photons from $\Sigma^{0}$ decay are emitted at large enough angles to facilitate their detection. The apparatus has been

\#1 For a review of polarization in inclusive production of hyperons see ref. [2] 
described previously [3]. A schematic view of the apparatus is shown in fig. 1. A secondary beam of particles was produced by steering $28.5 \mathrm{GeV} / \mathrm{c}$ protons from the AGS onto a $0.25 \times 0.25 \times 15.0 \mathrm{~cm}(0.50$ interaction length, 0.43 radiation length) $B e$ target. From this secondary beam a $3.1 \mathrm{msrad}$ solid angle neutral beam emerged from a $1.8 \mathrm{~m}$ long collimator embedded in a $3.71 \mathrm{Tm}$ dipole magnet called the sweeper magnet. The axis of the collimator, defining the $z$ axis of the spectrometer coordinate system, was set at a $4^{\circ}$ angle to the incident proton beam in the horizontal plane. The $y$ axis lay in the vertical direction and the $\boldsymbol{x}$ axis at beam left. The sweeper magnetic field was along the $\boldsymbol{x}$ axis. The field had two functions: to sweep out unwanted charged particles, and to precess the spins of the $\Lambda$ hyperons traversing the neutral beam collimator. By periodic reversals of the field, biases in the polarization measurement were controlled.

The particle detector was located downstream from the sweeper magnet, following a $1.65 \mathrm{~m} \mathrm{He}$ filled region. The $\Lambda$ momentum was measured through its decay products, the proton and pion, using a magnetic spectrometer consisting of two analyzing magnets. D6 set at $0.324 \mathrm{Tm}$ for the momentum analysis of the pion, and D7 set at $2.72 \mathrm{Tm}$ for the momentum analysis of the proton. Charged particle tracks were measured using three banks of spark chambers. A lead-glass calorimeter set below the aperture of the rear spectrometer magnet determined the $\gamma$ energy and position. By placing the calorimeter out of the production plane, $\Sigma^{0}$ decays were selected which transfer a large fraction of their polarization to the $\Lambda$. The calorimeter was an array of 84 $6.35 \times 6.35 \times 6.35 \times 58.4 \mathrm{~cm} \mathrm{SF-2}$ lead-glass blocks longitudinal to the neutral beam covering a solid angle of $2.61 \mathrm{msrad}$ or $84 \%$ of the total solid angle made available to the $\gamma$ by the collimator aperture. Each block was calibrated in an electron test beam before and after the experiment.

The $\Sigma^{0}$ trigger was formed by requiring the coincidence of a $\Lambda$ trigger with a $\gamma$ trigger. The $\Lambda$ trigger required a high-momentum positively charged particle passing through both spectrometer magnets in coincidence with a second less energetic charged particle passing through the upstream D6 magnet. Triggers from $\Lambda$ decays inside the collimator were prevented by a scintillation counter veto at the exit of the sweeper magnet. The $\gamma$ trigger was the linear sum of the energy deposited in the lead-glass array with a $0.5 \mathrm{GeV}$ threshold. Triggers from charged particles striking the calorimeter were eliminated by a veto counter in front of the array. The event trigger was the logical OR of the $\Sigma^{0}$ trigger and the $\Lambda$ trigger prescaled by 64 . On average $6 \times 10^{8}$ protons in a 1.2 $s$ spill were incident on target producing 18 triggers of which two thirds were $\Sigma^{0}$ triggers. The proton beam intensity was kept at a level compatible with high, stable spark chamber efficiency. Two tapes of approximately 30000 events each required about four hours after which the sweeper polarity was reversed and the process repeated. Approximately $5 \times 10^{6} \Sigma^{0}$ triggers and $1.3 \times 10^{6} \Lambda$ triggers were recorded on magnetic tape.

The data for each sweeper polarity were analyzed separately $\# 2$. Good $\Lambda$ events were selected by requiring two charged particle tracks of opposite sign ema-

\#2 For a more detailed discussion of the analysis procedures see ref. [4]
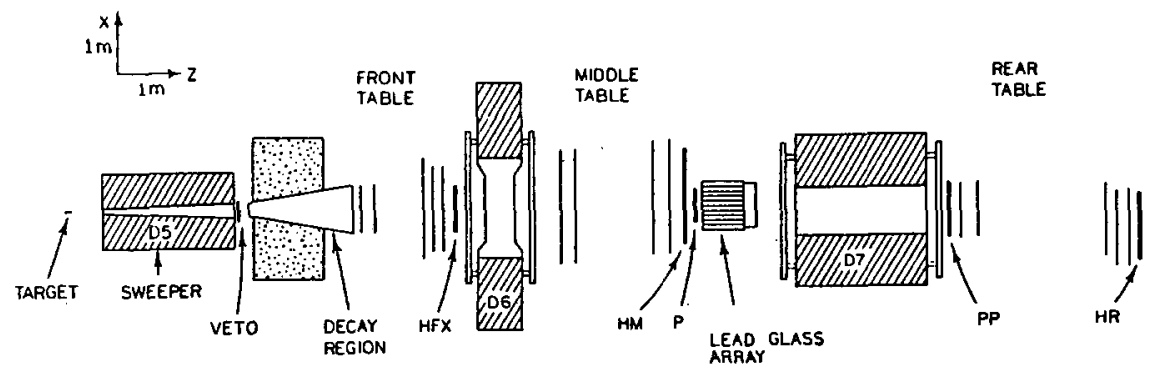

Fig. 1. Plan view of the apparatus. The $28.5 \mathrm{GeV} / \mathrm{c}$ proton beam impacted the target at $4^{\circ}$ to the collimator axis. Magnet D5 removed charged particles to form a neutral beam. The charged products from $\Lambda$ decay were detected with 28 planes of spark chambers situated in three tables. Hodoscope arrays HFX, HM, and HR, and scintillation counters $P$, PP, and VETO were used to form the $\Lambda$ trigger. 


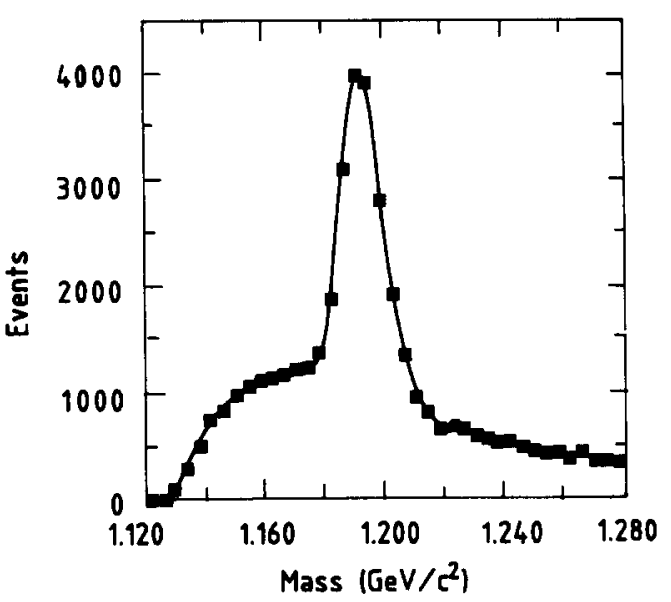

Fig. 2. The $\Lambda \gamma$ invariant mass.

nating from a common vertex with their vector momentum sum pointing back to the target. $A \Lambda$ was defined to be an event within $5 \sigma$, or $\pm 10 \mathrm{MeV} / c^{2}$, of the central value of the proton-pion invariant mass. Background was less than 1\%. After these requirements $178670 \Sigma^{0}$ triggers and $95255 \Lambda$ triggers remained. For the $\Sigma^{0}$ trigger sample the $\gamma$ energy was determined by first finding the lead-glass block with the maximum amount of energy in the array and then summing its energy with that of the eight surrounding blocks. Showers with maxima in an edge block were discarded as were showers out of time with the $\Lambda$ trigger. To track the variation of the lead-glass calibration constants during the experiment, run by run calibrations were done by constraining well defined $\Sigma^{0}$ topologies to the $\Sigma^{0}$ mass. The resultant invariant mass distribution is shown in fig. 2. The $\gamma$ energy resolution is $12 \%$ (RMS) for an average $\gamma$ energy of $1.5 \mathrm{GeV}$. Measurement of the polarization of the $\Lambda$ data provided an excellent check of systematic effects as it has been previously measured at this energy. Parity conservation constrained the initial $\Lambda$ polarization to lie in the $\pm y$ direction. The sweeper magnetic field then precessed the $\Lambda$ spin by an angle $\Omega=41.6^{\circ} \pm 0.5^{\circ}$ about the $x$ axis, the direction dependent upon the sign of the field. The polarization was found through the measurement of the asymmetry in the proton distribution in the $\Lambda$ rest frame along $z$ axis. The measured asymmetry is the sum of a term reversing sign with polarity change, $\alpha_{\Lambda} P_{\Lambda} \sin \Omega$, and a term representing apparatus-

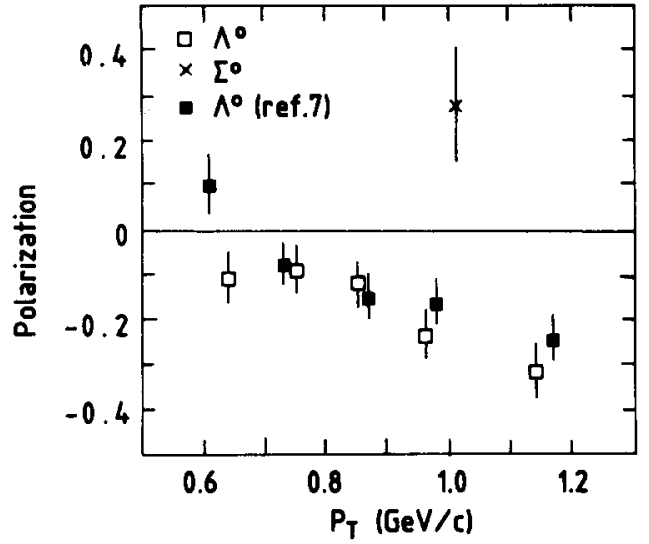

Fig. 3. The $\Sigma^{0}$ polarization and the $\Lambda$ polarization as a function of $p^{\mathrm{t}}$. Also shown is the $A$ polarization from ref. [7], from a subset of their data using a Be target and $28.5 \mathrm{GeV} / c$ incident protons.

induced asymmetries ("biases"). Biases were eliminated by subtracting the opposing polarity $z$ asymmetries. The probability of the proton being found in a particular element of phase space $\mathrm{d}\left(\cos \theta_{z}\right)$ is given by

$\mathrm{d} N / \mathrm{d}\left(\cos \theta_{z}\right)=A\left(\cos \theta_{z}\right)\left(1+\alpha_{\Lambda} P_{\Lambda z} \cos \theta_{z}\right) / 2$,

where $\theta_{z}$ is the angle the proton makes with respect to the $z$ axis, $A\left(\cos \theta_{z}\right)$ is the apparatus acceptance, and $\alpha_{\Lambda}=+0.642 \pm 0.013$ [5]. The acceptance was determined using a Monte Carlo hybrid technique [6] in which all the kinematic parameters of the simulated $\Lambda$ and its decay, except the $\cos \theta_{z}$ of the proton, are taken from the real events being analyzed. A least squares fit of the Monte Carlo $\cos \theta_{z}$ distribution to the real data was used to find the best value of $\alpha_{\Lambda} P_{z}$. The $\chi^{2}$ averaged over all analysis axes and $p_{t}$ bins was 20.1 for 18 degrees of freedom. The resultant polarization of 53000 events, plotted as a function of $p_{\mathrm{t}}$ is shown in fig. 3. The errors are statistical. Shown for comparison are the results of Ray. chaudhuri et al., [7] which was done ate the same energy and with similar apparatus. Our results agree with these as well as other results at different energies.

The polarization of $\Sigma^{0}$ must be measured through the daughter $\Lambda$ polarization. Parity conservation requires that, if polarized, the $\Sigma^{0}$ be produced with polarization along the $y$ axis. The short lifetime of the $\Sigma^{0}$ precludes any possibility of precession of its polarization before decay where it transfers a fraction of its polarization, 
$\boldsymbol{P}_{\Lambda}=-\left(\boldsymbol{P}_{\Sigma} \cdot \boldsymbol{\lambda}\right) \boldsymbol{\Lambda}$,

to the daughter [8]. Here $\boldsymbol{\lambda}$ is a unit vector in the direction of the $\Lambda$ momentum in the rest frame of the $\Sigma^{0}$. The $\Lambda$, as it traverses the magnetic field of the sweeper magnet, has its polarization precessed by an amount $\Omega=41.6^{\circ} \sin \delta$ about the $x$ axis, the direction dependent upon the sign of the magnetic field, where $\delta$ is the angle the $\Lambda$ polarization makes with the sweeper magnetic field. The components of the precessed $\Lambda$ polarization in terms of the $\Sigma^{0}$ polarization are

$P_{\Lambda x}=-\left(P_{\Sigma} \Lambda_{y}\right) A_{x}$,

$P_{\Lambda y}=-\left(P_{\Sigma} \Lambda_{y}\right)\left(\Lambda_{y} \cos \Omega \pm \Lambda_{z} \sin \Omega\right)$,

$P_{\Lambda z}=-\left(P_{\Sigma} A_{y}\right)\left( \pm \Lambda_{y} \sin \Omega+\Lambda_{z} \sin \Omega\right)$.

Measurement of each component of the $\Lambda$ polarization provides a separate measurement of the sign and the magnitude of the $\Sigma^{0}$ polarization diluted by a factor (analyzing power) dependent on the $\Lambda$ direction cosines in the $\Sigma^{0}$ rest frame as well as the precession angle $\Omega$.

The background in the $\Sigma^{0}$ data sample could not be ignored. The composition of the background was studied extensively and found to be $\Lambda$ 's with random $\gamma$ 's in the calorimeter. Analysis of the polarization of the background indeed showed the same polarization to that found from the $\Lambda$ data sample. The background has several adverse effects. The most serious consequence is that the determination of the $\Lambda$ momentum in the $\Sigma^{0}$ rest frame will obviously be incorrect as the very existence of a $\Sigma^{0}$ rest frame is meaningless for such events. This produces a distortion in the proton $\cos \theta$ distribution since to find the proton momentum direction cosines in the $A$ rest frame both the $A$ and the proton momentum must first be boosted into the $\Sigma^{0}$ rest frame before the proton is boosted into the $\Lambda$ rest frame. The solution to this problem is to boost directly the proton into the $\Lambda$ rest frame. This leads to an unwanted Lorentz rotation, but it is small and may be neglected. Monte Carlo studies show the average rotation is only $0.19^{\circ}$ with extremes of $\pm 1.50^{\circ}$. The determination of the analyzing power also requires the knowledge of the $\Lambda$ direction cosines in the $\Sigma^{0}$ rest frame. The effect of the background on this quantity was calculated by a Monte Carlo method as well as from the data. The difference between the two is less than $2 \%$. Finally, the background affects the measured asymmetry by directly contributing to the $\cos \theta$ distribution of the proton.

Knowledge that the background consists of prompt $\Lambda$ events allows one to write the measured asymmetry as the sum of three terms: an unknown asymmetry due to the $\Sigma^{0}$ events, a known asymmetry due to the background $\Lambda$ events, and an unknown asymmetry due to bias. The bias in the data was eliminated in a manner similar to that done for the $\Lambda$ polarization analysis. Were the acceptance of the $\Sigma^{0}$ decay products perfect, the average $\Lambda$ polarization before precession would be zero along the $x$ and $z$ axes and $-P_{\Sigma} / 3$ along the $y$ axis. The limited acceptance of the lead-glass array, $9 \%$ of $4 \pi$ at $16.5 \mathrm{GeV} / c$ (the average $\Sigma^{0}$ momentum), restricted the initial $\Lambda$ polarization to be constrained along a narrow cone centered in the $y z$ plane at an angle of $41^{\circ}$ to the $y$ axis. The average precession angle $\Omega$ from this initial value was $40^{\circ}$ for both sweeper polarities, resulting in a component of polarization perpendicular to the initial polarization which reversed sign with polarity change. It is from this component that the measurement of the polarization is made. The background $\Lambda$ polarization, on the other hand, is initially along $y$ and precessed to $\pm 42^{\circ}$ in the $y z$ plane according to the polarity of the sweeper magnet.

The Monte Carlo hybrid analysis method was employed to measure the proton asymmetry, giving a $\chi^{2}$ of 17.8 for 18 degrees of freedom. A total of 16000 events, equally divided between the two sweeper polarities, were analyzed. The prompt $\Lambda$ background was $31.5 \%$. The $\Sigma^{0}$ polarization is +0.28 \pm 0.13 at an average $p_{\mathrm{t}}=1.01 \mathrm{GeV} / c$ and $x_{\mathrm{f}}=0.60$. The error is statistical, but includes errors in the determination of the background fraction and the measurement errors for the prompt $\Lambda$ asymmetry. The background contribution to the measured asymmetry is less than one third. Systematic effects were studied as well as the effects of various cuts, none of which produce any statistically significant change in the result. The result is plotted in fig. 3 .

Despite the large amount of experimental data and the tantalizing behavior the data exhibit, a convincing theoretical explanation of inclusive polarization is still to come. Various phenomenogical models have 
been proposed [9] to explain inclusive polarization, all of which exploit the success the SU(6) static quark model has enjoyed in describing the spin structure of the hyperons. All of these models predict the $\Sigma^{0}$ polarization to be opposite that of the $\Lambda$. Our result is consistent with these models. Whereas the spin of the $\Lambda$ is entirely determined by the spin of the s quark, the $\Sigma^{0}$ has its spectator $u$ and d quarks in a spin triplet state which can contribute along with the s quark to the overall spin. If we assume that the polarization of the $\Sigma^{0}$ is due solely to the polarization of the $s$ quark, the $\Sigma^{0}$ should be produced with $-1 / 3$ the polarization of the $\Lambda$. The observed $\Sigma^{0}$ polarization is comparable to both $\Sigma^{+}$[10] and $\Sigma^{-}$[11] polarizations measured at Fermilab.

We remark that all of the $\Lambda$ polarization results previously reported are an underestimate of the true $\Lambda$ polarization as these experiments do not distinguish $\Lambda$ 's from $\Sigma^{0}$ decay from directly produced $\Sigma$ 's. We have measured the ratio of $\Sigma^{0}$ to $\Lambda$ inclusive production and find it to be 0.278 independent of $p_{\mathrm{t}}[3]$. Assuming the kinematic behaviour of the $\Sigma^{0}$ polarization is similar to that of the measured $\Lambda$ polarization these results imply that the actual prompt $\Lambda$ polarization is greater by about $25 \%$.

We would like to thank the Staff of the AGS Department of Brookhaven National Laboratory for their invaluable assistance. We are indebted to $R$.
Morrison of the University of California at Santa Barbara for the loan of some lead-glass blocks. The work was supported in part by the US National-Science Foundation and the US Department of Energy.

\section{References}

[1] G. Bunce et al., Phys. Rev. Lett. 36 (1976) 1113.

[2] L.G. Pondrom, Phys. Rep. 122 (1985) 57.

[3] M. Sullivan et al., Phys. Lett. B 142 (1984) 451; M. Sullivan, Measurement of the ratio of $\Sigma^{0}$ to $\Lambda$ inclusive production from $28.5 \mathrm{GeV} / c$ protons on beryllium, $\mathrm{Ph}$. D. Thesis, University of Massachusetts, Amherst, MA (September 1982), unpublished.

[4] E.C. Dukes, Measurement of the $\Lambda$ and $\Sigma^{0}$ polarizations in inclusive production from $28.5 \mathrm{GeV} / \mathrm{c}$ protons on beryllium, $\mathrm{Ph}$. D. Thesis, University of Michigan, Ann Arbor, MI (October 1984), unpublished.

[5] Particle Data Group, G.C. Wohl et al., Rev. Mod. Phys. 56 (1984) S1.

[6] G. Bunce, Nucl. Instrum. Methods 172 (1980) 553.

[7] K. Raychaudhuri et al., Phys. Lett B 90 (1980) 319.

[8] R. Gatto, Phys. Rev. 109 (1958) 610.

[9] K. Heller et al., Phys. Rev. Lett. 41 (1978) 607; 45 (1980) 1043 (E);

B. Andersson et al., Phys. Lett. B 85 (1979) 417;

T.A. DeGrand and H.I. Miettinen, Phys. Rev. D 24 (1981) 2419; D 31 (1985) 661(E).

[10] C. Wilkinson et al., Phys. Rev. Lett. 46 (1981) 803; C. Ankenbrandt et al., Phys. Rev. Lett. 51 (1983) 863;

C. Wilkinson et al., Phys. Rev. Lett. 58 (1987) 855.

[11] L. Deck et al., Phys. Rev. D 28 (1983) 1; Y.W. Wah et al., Phys. Rev. Lett 55 (1985) 2551. 